\title{
Variación morfométrica y geográfica entre poblaciones de Hypsiboas cordobae (Anura: Hylidae) en Argentina
}

\author{
Mariana Baraquet ${ }^{1,2}$, Pablo Raul Grenat ${ }^{1,2}$, Nancy Edith Salas ${ }^{1}$ y Adolfo Ludovico Martino ${ }^{1}$ \\ ${ }^{1}$ Ecología, Departamento de Ciencias Naturales, Facultad de Ciencias Exactas, Físico-Químicas y Naturales, Universidad Nacional de Río Cuarto, \\ Ruta Nacional №36-Km 601, Córdoba, Argentina; mbaraquet@exa.unrc.edu.ar; nsalas@exa.unrc.edu.ar; amartino@exa.unrc.edu.ar \\ ${ }^{2}$ CONICET, Consejo Nacional de Investigaciones Científicas y Técnicas, Av. Rivadavia 1917 C1033AAJ CABA, Argentina
}

Recibido 26-III-2012 Corregido 31-V-2012 Aceptado 26-VII-2012

\begin{abstract}
Morphometric differentiation and geographic variation among populations of Hypsiboas cordobae (Anura: Hylidae). Intraspecific phenotypic differentiation among geographically separated populations is common in anurans. We performed morphometric analyses of the endemic frog Hypsiboas cordobae in order to assess the geographic variation of morphometric variables along of distribution of the species. We measured 15 morphometric variables on 86 individuals from six localities of Córdoba and San Luís provinces. We found sexual dimorphism in ten variables. The comparative analysis showed significant differences between populations and discriminant analyses showed high percentages of classification (87,50\%). Eight morphometric variables showed positive correlation with latitude; and ten variables showed positive correlation with altitude. However, the correlations between morphometric distances and latitudinal/altitudinal were not significant. This geographic variation in the morphometry of the species may be due to environmental differences, or may be due to the age of individuals. Therefore, these data could be supplemented by studies directly related to the environmental conditions of each locality, as well as skeleton-chronological study to determine individual age.
\end{abstract}

\section{KEY WORDS}

Hypsiboas cordobae, morphological measures, geographic variation, Argentina.

\section{RESUMEN}

Las diferencias fenotípicas intraespecíficas entre poblaciones separadas geográficamente es común entre los anfibios anuros. En el presente trabajo se realiza una caracterización morfométrica de Hypsiboas cordobae, con el objetivo de estudiar la variación geográfica a lo largo de la distribución de la especie. Se midieron 15 variables un total de 86 individuos, pertenecientes a seis localidades de las provincias de Córdoba y San Luís. Se observó dimorfismo sexual en diez de las variables. El análisis comparativo mostró diferencias significativas entre poblaciones, y el análisis discriminante mostró altos porcentajes de clasificación (87,50\%). Ocho variables morfométricas mostraron relación significativa y positiva con la latitud; mientras que diez variables mostraron relación significativa y positica con la altitud. Sin embargo, en ambos casos las correlaciones entre las distancias morfométricas y las latitudinales/altitudinales no resultaron significativas (Test de Mantel). Esta variación geográfica en la morfometría de la especie puede deberse a diferencias ambientales, o podrían deberse también a la edad de los individuos. Por lo tanto, estos datos podían ser complementados con estudios relacionados directamente a las condiciones ambientales de cada localidad, como así también estudios de esqueletocronología que permitan determinar la edad de los individuos.

\section{PALABRAS CLAVE}

Hypsiboas cordobae, medidas morfométricas, variación geográfica, Argentina.
La morfometría es utilizada en la descripción cuantitativa, análisis, interpretación y variación de las formas. Así, estudios en sistemática basados en la morfología de los organismos requieren técnicas de descripción y comparación de la estructura de las formas (Rohlf 1990, Bernal \& Clavijo 2009). Debido a la importancia del tamaño corporal y de estructuras corporales en la ecología, en las historias de vida y en el éxito reproductivo de la mayoría de los organismos, los patrones interespecíficos de variación en el tamaño corporal pueden reflejar patrones de divergencia adaptativa (Fairbairn 1997).

En anuros, estudios basados en características morfológicas han permitido explicar relaciones evolutivas, 
taxonómicas y filogenéticas entre ellos, por ejemplo, descartando o aceptando diferencias entre grupos de individuos que ocupaban un nivel taxonómico incierto (Barrio 1965, Cei 1980, Heyer et al. 1990, Arikan et al. 1998, Castellano et al. 1998, Castellano \& Giacoma 2000, Maneyro \& Arrieta 2000, Lobo 2001; Lobo \& Abdala 2001, 2002, Sinsch et al. 2002, Oliver \& Lobo 2002, Méndez et al. 2004, Arroyo et al. 2005, Fabrezi 2006). Debido a la limitada capacidad de dispersión y filopatría en anfibios (Seppä \& Laurila 1999; Lampert et al 2003), es frecuente que poblaciones separadas geográficamente presenten diferencias intraespecíficas en sus estructuras morfológicas, particularmente en el tamaño corporal, generadas por una acumulación de diferencias genéticas y morfológicas debido a este aislamiento geográfico (Castellano et al. 2000, Laugen et al. 2002, Schäuble 2004).

El género Hypsiboas (Wagler, 1830) se encuentra representado por 84 especies de ranas, distribuidas en siete grupos. El grupo Hypsiboas pulchellus cuenta con 36 especies distribuidas en las regiones Andinas de Perú, Bolivia y Argentina, entre los 500 a 3416 msnm, así como también en regiones montañosas de Argentina, Paraguay, Brasil y Uruguay (Köhler et al. 2010, Lehr et al. 2010, Frost 2011, Lehr et al. 2011). Dentro de este grupo se encuentra la especie Hypsiboas cordobae (Barrio 1965), reportada para la zona central de Argentina (Barrio 1965, Cei 1980, Gallardo 1987, Bridarolli \& di Tada 1994). Esta especie presenta una distribución limitada y variable a lo largo del rango altitudinal, distribuyéndose en las sierras de las provincias de Córdoba y San Luís, Argentina, y desconociéndose con precisión los límites de su distribución particularmente en la zona de contacto con H. pulchellus (Barrio 1965, Cei 1980, Gallardo 1987). Por su parte, esta especie se encuentra dentro de la categoría de datos deficientes de acuerdo con la Lista Roja de las Especies de la UICN, resaltando la importancia y necesidad de mas estudios sobre su ecología y distribución.

A pesar de estudios que describen la morfometría de H. cordobae (Barrio 1965, Cei 1980, Gallardo 1987), la variación morfométrica intraespecífica entre diferentes poblaciones no ha sido estudiada en esta especie. En este estudio se realiza una caracterización morfométrica de $H$. cordobae en general, pero también caracterizando individuos machos y hembras. Además, se estudia la variación geográfica de la morfometría de H. cordobae en seis poblaciones a lo largo del rango de distribución de la especie, tomando en cuenta los diferentes niveles latitudinales $y$ altitudinales de las poblaciones estudiadas.

\section{METODOLOGÍA}

Se colectaron 86 individuos de $H$. cordobae a lo largo de un gradiente latitudinal de seis localidades de las provincias de Córdoba y San Luis, Argentina, entre Mayo de 2006 y Septiembre de 2011. Las localidades fueron: Achiras (808msnm, 3309'28,64" S-64058'55,13"W); Las Guindas (Alpa Corral, 930msnm, 32035'35,22" S-

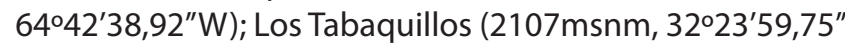
S-64055'33,69"W); Pampa de Achala (2150msnm, 31049'41,8" S-64'51'44,9"W); Los Linderos (2310msnm, $32^{\circ} 00^{\prime} 54,0^{\prime \prime}$ S-64056'42,97"W); La Carolina (1634msnm, $32^{\circ} 48^{\prime} 43,94^{\prime \prime}$ S-660 $05^{\prime} 48,15^{\prime \prime}$ W) (Fig. 1.).

La morfometría de los ejemplares capturados se caracterizó en base a 15 variables morfométricas (Cei 1980, Heyer et al. 1990, Martino \& Sinsch 2002) realizadas con los organismos en vida. Las medidas fueron realizadas mediante el uso de calibre digital Mahr $16 \mathrm{ES}( \pm 0,01 \mathrm{~mm})$. Las mediciones fueron: (1) longitud hocico-cloaca (LHA); (2) anchura máxima de la cabeza (HW); (3) largo de la cabeza (LC); (4) distancia hocico-ojo (HO); (5) distancia

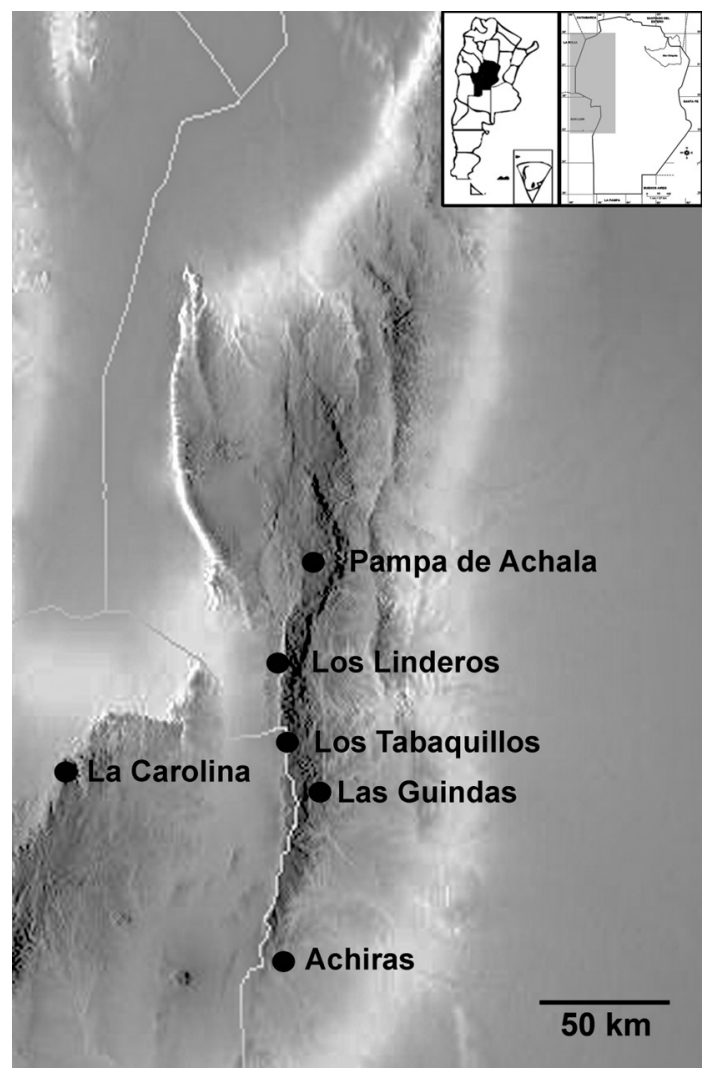

FIG. 1. Mapa que muestra las seis localidades en donde fueron colectados los individuos de $\mathrm{H}$. cordobae. 
internarinas (IN); (6) distancia interocular (IO); (7) distancia ojo-narina (ON); (8) distancia rostro-narina (RN); (9) diámetro del ojo (DO); (10) largo del brazo (LB); (11) largo del tercer dígito (lado interno) anterior (TF); (12) largo del fémur (LF); (13) largo de la tibia (LT); (14) largo de la pata (LP); (15) largo del cuarto dígito (lado interno) posterior (CT).

Se obtuvieron promedios, desviaciones, y medidas máximas y mínimas de cada variable; una medida general para la especie y para cada población. Se probó la normalidad de los datos en las 15 variables mediante la prueba de Shapiro-Wilks y se realizaron comparaciones entre machos y hembras respecto a la morfometría, mediante una prueba de análisis de varianza (ANDEVA) en los datos con distribución normal y mediante ANDEVA de Kruskal-Wallis en los casos en que la normalidad fue rechazada.

Para los análisis comparativos entre poblaciones, se probó la normalidad de los datos mediante la prueba de Shapiro-Wilks; se realizaron pruebas de ANDEVA en los datos con distribución normal y Kruskal-Wallis en los casos en que la normalidad fue rechazada. En el caso en que los resultados mostraron diferencias significativas se realizó un test a posteriori Tukey HSD para determinar diferencias entre poblaciones. Luego se realizó un Análisis Discriminante para obtener una medida adicional de la diferenciación morfológica entre poblaciones (Sinsch \& Schneider 1999).

Se realizaron regresiones simples entre las variables morfométricas, la latitud y altitud de cada localidad. A partir del análisis discriminante realizado se calcularon las diferencias morfométricas entre las seis poblaciones con las distancias de Mahalanobis. Para evaluar la asociación entre las distancias geográficas (en kilómetros) y las distancias morfométricas (distancias de Mahalanobis) se realizó el test de Mantel utilizando MANTEL para Windows 1.18 (Cavalcanti 2005). Todos los análisis estadísticos fueron realizados con el programa Statgraphics Plus 5.0.

\section{RESULTADOS}

Los machos adultos de $\mathrm{H}$. cordobae presentaron una longitud total promedio de 50,02 mm $(41,30-61,23$ ठै), mientras que las hembras fue de $54,50 \mathrm{~mm}(41,1-61,87$ + $)$ (Cuadro 1). El largo promedio de la cabeza $(15,72 \mathrm{~mm})$

CUADRO 1

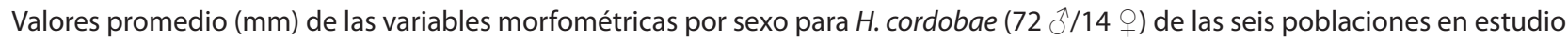

\begin{tabular}{|c|c|c|c|c|c|c|c|c|c|c|c|c|}
\hline & \multicolumn{2}{|c|}{ La Carolina } & \multicolumn{2}{|c|}{ Pampa de Achala } & \multicolumn{2}{|c|}{ Achiras } & \multicolumn{2}{|c|}{ Las Guindas } & \multicolumn{2}{|c|}{ Los Tabaquillos } & \multicolumn{2}{|c|}{ Los Linderos } \\
\hline & $14 \hat{\sigma}$ & 1 운 & $10 \widehat{\jmath}$ & 6 우 & $10 \lesssim$ & 19 & $25 \widehat{\sigma}$ & 3 우 & 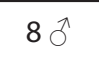 & 3 웅 & $5 \hat{\sigma}$ & q \\
\hline SVL & 51,25 & 53,61 & 49,56 & 57,21 & 53,43 & 55,08 & 46,78 & 46,14 & 50,56 & 57,53 & 56,99 & - \\
\hline HW & 15,21 & 16,55 & 14,38 & 16,18 & 15,61 & 15,86 & 13,45 & 13,62 & 15,55 & 16,97 & 15,91 & - \\
\hline LC & 16,21 & 16,1 & 15,81 & 17,91 & 16,61 & 16,61 & 14,53 & 15,19 & 16,35 & 18,58 & 17,36 & - \\
\hline HO & 6,94 & 7,43 & 6,33 & 6,99 & 7,23 & 7,65 & 6,04 & 6,72 & 6,89 & 8,00 & 7,42 & - \\
\hline IN & 4,19 & 4,78 & 4,20 & 4,53 & 4,43 & 4,17 & 3,68 & 3,81 & 4,28 & 4,89 & 4,55 & - \\
\hline 10 & 5,60 & 5,29 & 5,07 & 5,80 & 5,73 & 5,8 & 5,45 & 5,51 & 5,47 & 6,00 & 5,80 & - \\
\hline ON & 4,26 & 3,65 & 4,15 & 4,69 & 4,22 & 4,41 & 3,51 & 3,41 & 4,03 & 4,27 & 4,47 & - \\
\hline RN & 3,32 & 3,33 & 2,79 & 2,80 & 3,40 & 3,39 & 3,17 & 3,22 & 3,43 & 4,19 & 3,29 & - \\
\hline DO & 4,81 & 5,11 & 5,14 & 5,59 & 5,52 & 5,45 & 5,04 & 5,25 & 5,03 & 5,66 & 5,86 & - \\
\hline LB & 24,64 & 25,96 & 24,57 & 26,98 & 25,91 & 25,06 & 21,94 & 21,41 & 23,68 & 26,89 & 27,84 & - \\
\hline TF & 10,37 & 11,06 & 10,78 & 11,81 & 11,05 & 11,72 & 8,94 & 7,94 & 9,88 & 9,91 & 12,49 & - \\
\hline LF & 26,51 & 28,87 & 25,97 & 29,67 & 28,18 & 24,11 & 23,04 & 24,52 & 26,36 & 29,05 & 28,75 & - \\
\hline LT & 25,96 & 27,68 & 25,08 & 28,59 & 28,02 & 37,39 & 23,70 & 24,39 & 25,47 & 27,88 & 27,81 & - \\
\hline LP & 36,87 & 33,12 & 36,13 & 40,84 & 38,49 & 37,07 & 32,97 & 33,61 & 36,20 & 39,79 & 37,55 & - \\
\hline CT & 14,64 & 14,1 & 16,30 & 17,91 & 14,80 & 15,9 & 12,29 & 13,36 & 13,61 & 13,46 & 17,12 & - \\
\hline
\end{tabular}


corresponde al $34,43 \%$ de la longitud total, y es algo mayor a su ancho (14,63 mm); mostrando un índice cefálico (ancho de la cabeza/largo de la cabeza) de 0,93. Las hembras presentaron en promedio LHA mayor a los de los machos (54,50 mm).

La mayoría de las variables (10) presentaron diferencias morfometricas entre machos y hembras, mientras que las variables distancia interocular (IO), distancia ojo-narina $(\mathrm{ON})$, distancia rostro-narina (RN), en el Largo del $3^{\mathrm{er}}$ digito anterior (TF) y Largo del $4^{\text {to }}$ dígito posterior (CT) no mostraron diferencias (Cuadro 2).

Para la comparación entre las distintas poblaciones de H. cordobae, se utilizaron únicamente datos de los machos, debido al bajo número de hembras capturadas. Todas las variables morfométricas mostraron diferencias significativas (ANDEVA y ANDEVA de Kruskal-Wallis, $\mathrm{p}<0,05$ ). Los tests a posteriori Tukey HSD mostraron que para todas las variables, excepto en la IO, la población de Las Guindas se diferencia significativamente de las demás poblaciones, mostrando en todas las variables valores menores para esta población, mientras que en el caso de las demás variables, las diferencias entre poblaciones fue variable.

A partir del análisis discriminante se obtuvieron tres funciones altamente significativa $(p=0,0000 ; p=0,0000$; $p=0,0010$ ), la primera con un Eigenvalue de 4,31 explica el 57,09\% del total de la variación observada (Corr. Can. $=0,90101 ; \lambda$ de Wilks $=0,0217874)$; la segunda función con un Eigenvalue de 1,62 explica el 21,48\% del total de la variación observada (Corr. Can. $=0,78663 ; \lambda$ de Wilks $=$ $0,115778) ;$ y la tercera función discriminante con un Eigenvalue de 1,13 explica el 15,01\% del total de la variación observada (Corr. Can. $=0,72894 ; \lambda$ de Wilks =0,303713).

La función discriminante uno separa la población de Las Guindas de las demás poblaciones, siendo en esta
CUADRO 2

ANDEVA / Kruskal-Wallis de 15 variables morfométricas entre individuos machos y hembras de H. cordobae

\begin{tabular}{lcc}
\hline Variable & $\mathrm{p}$ & $\mathrm{F} \mathrm{o} \mathrm{H}$ \\
\hline SVL & 0,0005 & 12,91 \\
HW & 0,0031 & 9,27 \\
LC & 0,0007 & 12,52 \\
HO & 0,0046 & 8,46 \\
IN & 0,0125 & 6,52 \\
ON & 0,0882 & 2,98 \\
RN & 0,7243 & 0,13 \\
DO & 0,0079 & 7,42 \\
LB & 0,0239 & 5,29 \\
TF & 0,3633 & 0,84 \\
LF & 0,0022 & 9,98 \\
LT & 0,0003 & 14,18 \\
LP & 0,0094 & 7,06 \\
IO & 0.1881 & 12.91 \\
CT & 0.0646 & 3.68
\end{tabular}

Se indica el valor del p y el estadístico F del ANDEVA o $\mathrm{H}$ de Kruskal-Wallis para cada variable.

\section{CUADRO 3}

Porcentaje de observaciones correctamente clasificadas, en base a las funciones obtenidas a partir del análisis discriminante usando variables morfométricas de $H$. cordobae para las seis poblaciones estudiadas

\begin{tabular}{cccccccc}
\hline Grupo Actual & $\mathbf{n}$ & $\mathbf{1}$ & $\mathbf{2}$ & $\mathbf{3}$ & $\mathbf{4}$ & $\mathbf{5}$ & $\mathbf{6}$ \\
\hline $\mathbf{1}$ & 14 & $11(78,57 \%)$ & 0 & 0 & 0 & $2(14,29 \%)$ & $1(7,14 \%)$ \\
$\mathbf{2}$ & 10 & $1(10 \%)$ & $7(70 \%)$ & $2(20 \%)$ & 0 & 0 & 0 \\
$\mathbf{3}$ & 10 & $1(10 \%)$ & 0 & $9(90 \%)$ & 0 & 0 & 0 \\
$\mathbf{4}$ & 25 & 0 & 0 & 0 & $24(96 \%)$ & $1(4 \%)$ & 0 \\
$\mathbf{5}$ & 8 & $1(12.50 \%)$ & 0 & 0 & 0 & $7(87.50 \%)$ & 0 \\
$\mathbf{6}$ & 5 & 0 & 0 & 0 & 0 & 0 & $5(100 \%)$
\end{tabular}

(1) La Carolina; (2) Pampa de Achala; (3) Achiras; (4) Las Guindas; (5) Los Tabaquillos; (6) Los Linderos. 
función la variable de mayor peso el largo del fémur. La función discriminante dos separa el resto de las localidades, con el largo del tercer dígito anterior como variable de mayor peso (Fig. 2).

El porcentaje de individuos correctamente clasificados fue de $87,50 \%$. En la tabla de clasificación (Cuadro 3) se observa que el porcentaje de individuos correctamente clasificados fue alto, siendo igual o mayor al $70 \%$ en todos los casos.

Las regresiones entre cada variable morfométrica y las latitudes de cada población mostraron que de las 15 variables, ocho se relacionan significativamente con la latitud, y en todas las variables el coeficiente de correlación fue positivo (Cuadro 4).

El test de Mantel, utilizado para estimar la correlación entre las distancias latitudinales y las distancias morfométricas (distancias de Mahalanobis), mostró que las diferencias mofométricas no presentaron correlación significativa con las distancias geográficas $(p=0,8866$; $r=-0,311 ; t=-1,2084)$.

Luego se realizaron regresiones simples entre cada variable morfométrica y la altitud de cada población. De las 15 variables morfométricas, diez mostraron relación significativa con la altitud, y en todas el coeficiente de correlación fue positivo (Cuadro 4).

El test de Mantel, utilizado para estimar la correlación entre las distancias altitudinales (msnm) y las distancias morfométricas (distancias de Mahalanobis), mostró que las diferencias morfométricas no presentaron correlación significativa con las distancias geográficas $(p=0,9649 ; r=$ $-0,499 ; \mathrm{t}=-1,8112$ ).

\section{DISCUSIÓN}

El tamaño del cuerpo es un rasgo morfológico fundamental, importante en un contexto fisiológico, ecológico y social. La variación geográfica en las estructuras morfológicas, particularmente el tamaño corporal, es común en muchas especies (Schäuble 2004, Silva et al. 2008). Entre los anuros, los análisis de variación geográfica intraespecífica en morfología han revelado una amplia variación en el tamaño del cuerpo (Castellano et al. 2000; Schäuble 2004).

El estudio demostró que las poblaciones de $\mathrm{H}$. cordobae varían en su morfometría. Las diferencias fenotípicas intraespecíficas entre poblaciones separadas geográficamente es común, y si bien el determinante de esta diferenciación es aún discutido, puede ocurrir como respuesta directa a la heterogeneidad del medio ambiente (Laugen et al. 2002). Las variaciones morfológicas en el tamaño corporal de poblaciones de anfibios anuros puede deberse a diferencias ambientales, es decir, diferentes microhábitats pueden favorecer diferentes tipos morfológicos (Narins \& Smith 1986).

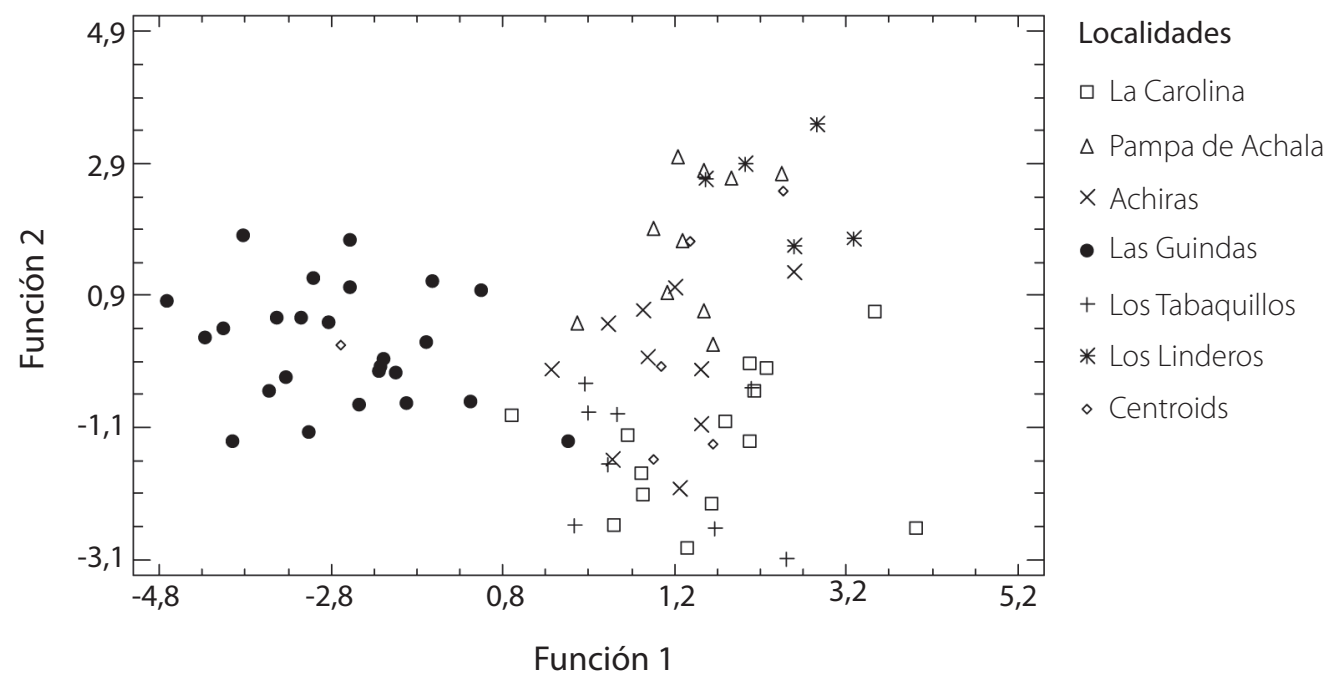

FIG. 2. Función 1 y Función 2 del Análisis Discriminante realizado sobre las variables morfométricas de H. cordobae para las seis poblaciones estudiadas. 


\section{CUADRO 4}

Valor de $p, r$ y $\mathrm{R}^{2}$ de las regresiones lineales entre los niveles latitudinales y altitudinales con cada una de las poblaciones estudiadas

\begin{tabular}{lcccccc}
\hline & \multicolumn{3}{c}{ Latitud } & \multicolumn{3}{c}{ Altitud } \\
\hline Variable & $\boldsymbol{p}$ & $\mathrm{r}$ & $\mathrm{R}^{2}$ & $\boldsymbol{p}$ & $\mathbf{r}$ & $\mathrm{R}^{2}$ \\
\hline LHA & $0,0077^{*}$ & 0,311852 & $9,72 \%$ & $0,0089^{*}$ & 0,306112 & $9,37 \%$, \\
HW & $0,0288^{*}$ & 0,257718 & $6,64 \%$ & $0,0020^{*}$ & 0,358691 & $12,87 \%$ \\
LC & 0,1360 & 0,177407 & $3,15 \%$ & $0,0012^{*}$ & 0,374233 & $14,00 \%$ \\
HO & $0,0021^{*}$ & 0,356189 & $12,68 \%$ & 0,0401 & 0,242505 & $5,88 \%$ \\
IN & 0,2552 & 0,135857 & $1,84 \%$ & $0,0015^{*}$ & 0,36691 & $13,46 \%$ \\
ON & 0,2274 & 0,144028 & $0,07 \%$ & $0,0002^{*}$ & 0,422561 & $17,85 \%$ \\
RN & $0,0102^{*}$ & 0,300864 & $9,05 \%$ & 0,4121 & $-0,0981366$ & $0,96 \%$ \\
DO & 0,2656 & 0,132932 & $1,77 \%$ & 0,8353 & 0,0264657 & $0,07 \%$ \\
LB & $0,0456^{*}$ & 0,23637 & $5,58 \%$ & $0,0016^{*}$ & 0,3656 & $13,37 \%$ \\
TF & 0,2482 & 0,137842 & $1,90 \%$ & $0,0003^{*}$ & 0,418737 & $17,53 \%$ \\
LF & $0,0217^{*}$ & 0,270305 & $7,30 \%$ & $0,0009^{*}$ & 0,381359 & $14,54 \%$ \\
LT & $0,0011^{*}$ & 0,377696 & $14,26 \%$ & 0,1545 & 0,169537 & $2,87 \%$ \\
LP & 0,0522 & 0,229768 & $5,28 \%$ & $0,0308^{*}$ & 0,254766 & $6,49 \%$ \\
IO & $0,0074^{*}$ & 0,3129 & $9,79 \%$ & 0,3880 & $-0,103277$ & 1,06 \\
CT & 0,6108 & $-0,0609861$ & $0,37 \%$ & $0,0000^{*}$ & 0,500389 & $25,03 \%$
\end{tabular}

*Variables que mostraron Relación significativa.

En este trabajo se observó un incremento en el tamaño corporal en función de la altitud, es decir mayor tamaño corporal a mayor nivel altitudinal. Debido a que los anfibios son animales ectotermos, la mayoría de sus aspectos fisiológicos y comportamentales están afectados por la temperatura (Wells 2007); por lo cual, esta relación entre el tamaño corporal y los niveles altitudinales de las localidades de muestreo pueden ser explicadas por las diferencias climáticas entre localidades. Varios estudios en animales poiquilotermos, han demostrado que las variaciones morfométricas son causadas por una combinación de factores genéticos y ambientales (Castellano \& Giacoma, 1998; Tryjanowski et al. 2006, Stock et al. 2008, Amor et al. 2011). Bickford et al. (2007) indican que condiciones ambientales extremas podrían imponer que la selección se establezca sobre la morfología, reduciendo o eliminando cambios morfológicos que pueden acompañar a la especiación. Mientras que Gould \& Johnston (1972), al referirse a la relación entre variables morfológicas y ambientales, indican que si la variación intraespecífica es resultado de la selección natural, se debe esperar una fuerte asociación entre la morfología y los factores ambientales que influyen en la adaptación; siendo esta correlación la principal prueba de selección en la variación geográfica.

Una de las tendencias ecogeográficas más intensamente estudiadas es la variación en el tamaño corporal a través de un gradiente ambiental (Angilletta \& Dunham 2003). Especies distribuidas en un amplio rango geográfico a menudo presentan clinas térmicas en correlación con el tamaño corporal, en donde los adultos de la mayoría de las especies presentan mayor tamaño en ambientes más fríos (Bernardo \& Agosta 2003; Angilletta et al. 2004). En este sentido, la regla de Bergmann establece que los individuos de las especies que habitan ambientes con clima relativamente fríos tienden a ser más grandes que aquellos que habitan ambientes más cálidos (Angilletta et al. 2004; de Queiroz \& Ashton 2004). Si bien esta regla está basada en organismos endotermos, también es aplicada en numerosas especies de ectotermos (de Queiroz \& Ashton 2004). Incluso, estudios de laboratorio han demostrado que una reducción de la temperatura del medio ambiente provoca un aumento en el tamaño adulto en la 
mayoría de ectotermos (Atkinson 1994, 1995; Atkinson et al. 2003). Algunas especies de anfibios muestran un mayor tamaño corporal en climas fríos que en climas cálidos; son considerablemente más grandes a niveles altitudinales mayores que sus congéneres en altitudes más bajas. Posiblemente esto se deba al mayor efecto fisiológico de las bajas temperaturas sobre el desarrollo, más que sobre el crecimiento, pudiendo a su vez retrasar la madurez sexual (Wells 2007).

Relacionado también al clima, la regla de Bergmann establece que el tamaño del cuerpo aumenta con la latitud (Schäuble 2004). En H. cordobae también se observó un incremento en el tamaño corporal en función de la latitud; es decir, una variación clinal de norte a sur de aumento en el SVL, lo cual puede ser explicado en relación a las cuestiones climáticas y la regla de Bergmann. Además, el test de Mantel no mostró relaciones significativas entre las distancias geografías y la variación morfométrica, ni entre la altitud y la variación morfométrica. Esto apoya el hecho de que las variaciones morfométricas se deban a los diferentes niveles latitudinales y altitudinales de las poblaciones en estudio, y no a las distancias entre pares de localidades.

Castellano \& Giacoma (2000) proponen una segunda explicación, argumentando que el tamaño corporal de los individuos puede ser reflejo de su edad. En anfibios anuros, el tamaño corporal de los adultos depende de numerosos factores, incluyendo el tiempo de metamorfosis, la tasa crecimiento antes y después de la madurez, la edad de madurez y la longevidad (Rosso et al. 2004); por lo cual, si bien se encontraron diferencias morfométricas entre las especies, están podrían deberse en parte a la edad de los individuos.

Nuestro trabajo en $H$. cordobae a lo largo de la distribución de las especies mostró diferencias entre las poblaciones estudiadas, lo que confirma la presencia de la variación geográfica en la morfometría de la especie. Sin embargo, estos datos podrían ser complementados con estudios relacionados directamente a las condiciones ambientales de cada localidad, como así también estudios de esqueleto cronología que permitan determinar la edad de los individuos, evaluando la influencia de este factor en la variación geográfica de la morfometría.

\section{AGRADECIMIENTOS}

El primer autor agradece al Consejo de Nacional de Investigaciones Científicas y Tecnológicas (CONICET) por las Becas de Posgrado otorgadas. A Julián Valetti por su valiosa ayuda en las salidas de muestreo a campo. A la Secretaria de Ciencia y Técnica (SECyT) de la Universidad
Nacional de Río Cuarto que financia el proyecto en el cual se enmarca el presente estudio (PPI 18/C350). El presente estudio fue autorizado por la Secretaría de Ambiente de la provincia de Córdoba.

\section{REFERENCIAS}

Amor, N., S., Farjallah, S., Ben Yakoub \& K., Ssaid. 2011. Morphological Variation of the African Green Toad, Bufo boulengeri (Amphibia: Anura) in Tunisia. Pa. J. Zool. 43: 921-926.

Angilletta, M.J. \& A.E., Dunham. 2003. The temperature-size rule in ectotherms: simple evolutionary explanations may not be general. Am. Nat. 162: 332-342.

Angilletta, M.J., T.D., Steury \& M.W., Sears 2004. Temperature, Growth Rate, and Body Size in Ectotherms: Fitting Pieces of a Life-History Puzzle. Integr. Comp. Biol. 44:498-509.

Arikan, H., K., Olgun, I.E., Çevic \& C.V., Tok. 1998. A Taxonomical Study on the Rana ridibunda PALLAS, 1771 (Anura: Ranidae) Population from Ivriz-Eregli (Konya). Tr. J. Zool. 22: 181-184.

Arroyo, S.B., P.M., Sánchez, M.P., Ramírez-Pinilla, H.A., Suárez \& D.R., Miranda-Esquivel. 2005. Morphometric analysis to differentiate taxonomically seven species of Eleutherodactylus (Amphibia: Anura: Leptodactylidae) from an Andean cloud forest of Colombia. Zootaxa 1018: 1-14.

Atkinson, D. 1994. Temperature and organism size a biological law for ectotherms? Adv. Ecol. Res. 25:1-58.

Atkinson, D. 1995. Effects of temperature on the size of aquatic ectotherms: Exceptions to the general rule. J. Therm. Biol. 20: 61-74.

Atkinson, D., B.J., Ciotti \& D.J.S., Montagnes. 2003. Protists decrease in size linearly with temperature: ca. $2.5 \% 8 C 21$. Proc. Royal Soc. 270: 2605-2611.

Barrio, A. 1965. Las subespecies de Hyla pulchella Duméril \& Bibron (Anura, Hylidae). Phycis, 69: 115-128.

Bernal, M.H. \& J.A., Clavijo 2009. An essay on precision in morphometric measurements in anurans: inter-individual, intra-individual and temporal comparisons. Zootaxa 2246: 32-44.

Bernardo, J. \& S.J., Agosta. 2003. Determinants of clinal variation in life history of dusky salamanders (Desmognathus ocoee): prey abundance and ecological limits on foraging time restrict opportunities for larval growth. Lon. J. Zool. 259: 411-421.

Bickford, D., D.J., Lohman, N.S., Sodhi, P.K.L., Ng, R., Meier, K., Winker, K.K., Ingram \& I., Das. 2007. Cryptic species as a window on diversity and conservation. Trends Ecol. Evol. 22: 148-155.

Bridarolli, M.E. \& I.E., di Tada. 1994. Biogeografía de los Anfibios Anuros de la Región Central de la República Argentina. Cuad. Herpetol. 1: 63-82. 
Cavalcanti, M.J. 2005. Mantel for windows, Version 1.18. (Consultado: 20 noviembre 2011, http://life.bio.sunysb.edu/ morph/ ).

Castellano S. \& C., Giacoma. 1998. Morphometric variation of the green toad, Bufo viridis, in Italy: a test of causation. J. Herpetol. 32: 540-550.

Castellano S., C., Giacoma, T., Dujsebayeva, G., Odierna \& E., Balletto. 1998. Morphometrical and acoustical comparison between diploid and tetraploid green toads. Biol. J. Linn. Soc. 63: 257-281.

Castellano, S. \& C., Giacoma. 2000. Morphometric and advertisement call geographic variation in polyploid green toads Biol. J. Linn. Soc. 70: 341-360.

Castellano, S., C., Giacoma \& T., Dujsebayeva. 2000. Morphometrical and advertisement call geographic variation in polyploid green toads. Biol. J. Linn. Soc. 70:341-360.

Cei, J.M. 1980. Amphibians of Argentina. Monitore Zool. Ital. (n.s.) Monogr. 2 1-609.

De Queiroz, A. \& K.G., Ashton. 2004. The phylogeny of a specieslevel tendency: species heritability and possible deep origins of Bergmann's rule in tetrapods. Evolution 58(8): 1674-1684.

Fabrezi, M. 2006. Morphological evolution of Ceratophryinae (Anura, Neobatrachia). J. Zool. Syst. Evol. Res. 44: 153-166.

Fairbairn, D.J. 1997. Allometry for sexual size dimorphism: Pattern and process in the coevolution of body size in males and females. Annual Review of Ecology and Systematics, 28: 659-687. Cuad. Herpetol. 17 (1-2): 119-125.

Frost, D.R. 2011. Amphibian Species of the World: an Online Reference Version 5.5. American Museum of Natural History, New York, USA. (Consultado: 15 diciembre 2011, http:// research.amnh.org/vz/herpetology/amphibia/).

Gallardo, J.M. 1987. Anfibios Argentinos. Guía para su identificación. Agropecuaria, Buenos Aires, Argentina 98: 81-82.

Gould, S.J. \& R.F., Johnston. 1972. Geographic Variation. Ann. Rev. Ecol. Syst. 3: 457-498.

Heyer, W.R., A.S., Rand, C.A., Gonçalves da Cruz, O.L., Peixoto \&. C.E., Nelson. 1990. Frogs of Boracéia. Arq. Zool. (São Paulo) $31: 231:-410$.

Köhler, J., D., Koscinski, J.M., Padial, J.C., Chaparro, P., Handford, S.C., Lougheed \& I., De la Riva. 2010. Systematics of Andean gladiator frogs of the Hypsiboas pulchellus species group (Anura, Hylidae). Zool. Scr. 39: 572-590.

Lampert, K.P., A.S., Rand, U.G., Mueller \& M.J., Ryan. 2003. Finescale genetic pattern and evidence for sex-biased dispersal in the túngara-frog, Physalaemus pustulosus. Mol. Ecol. 12: 3325-3334.

Laugen, A.T., A., Laurilla \& J., Merilä. 2002. Maternal and genetic contributions to geographical variation in Rana temporaria larval life-history traits. Biol. J. Linn. Soc. 76: 61-70.
Lehr, E., J., Faivovich \& K.H., Jungfer. 2010 A new Andean species of the Hypsiboas pulchellus group: adults, calls and phylogenetic relationships. Herpetologica 66: 296-307.

Lehr, E., J., Faivovich \& K.H., Jungfer. 2011 Description of the tadpoles of Hypsiboas aguilari and H. melanopleura (Anura: Hylidae: Hypsiboas pulchellus group). Salamandra 47:30-35.

Lobo, F. 2001. A phylogenetis analysis of lizards of the Liolaemus chilensis group (Iguania: Tropiduridae). Herpetol. J. 11: 137-150.

Lobo, F. \& C., Abdala. 2001. Variación morfológica en el esqueleto de Liolaemus (Iguania: Liolaemidae). Búsqueda y descripción de caracteres. Cuad. Herpetol. 15(2): 119-135.

Lobo, F. \& C., Abdala. 2002. La información cladística de un set de datos morfológicos en lagartos del género Liolaemus (Iguania: Liolaemidae). Búsqueda y descripción de caracteres. Cuad. Herpetol. 16: 37-150.

Maneyro, R. \& D., Arrieta. 2000. Reporte de variaciones morfológicas en ejemplares de Bufo arenarum Hensel 1867, con comentarios sobre el grupo Marinus. Bol Soc. Zool. Uruguay 12: 1-13.

Martino, A.L. \& U., Sinsch. 2002. Speciation by polyploidy in Odontophrynus americanus. Lon. J. Zool. 257: 67-81.

Méndez, M.A, E.R., Soto, C., Correa, A., Veloso, E., Vergara, M., Sallaberry, \& P., Iturra. 2004. Morphological and genetic differentiation among Chilean populations of Bufo spinulosus (Anura: Bufonidae). Rev. Chil. Hist. Nat. 77: 559-567.

Narins, P.M. \& S.L., Smith. 1986. Clinal variation in anuran advertisement calls: basis for acoustic isolation? Behav. Ecol. and Sociobiol. 19:135-141.

Oliver, I.M. \& F., Lobo. 2002. Una nueva especie de Liolaemus del grupo alticolor (Iguania: Liolaemidae) de la Puna salteña, Argentina. Cuad. Herpetol. 16: 47-64.

Rohlf, F.J. 1990. Morphometrics. Annu. Rev. Ecol. Syst. 21: 299-316.

Rosso, A.; S., Castellano \& C., Giacoma. 2004. Ecogeographic analysis of morphological and life-history variation in the Italian frog. Evol. Ecol. Res. 18: 303-321.

Schäuble, C.S. 2004. Variation in body size and sexual dimorphism across geographical and environmental space in the frogs Limnodynastes tasmaniensis and L. peronii. Biol. J. Linn. Soc. 82: 39-56.

Seppä, P. \& A., Laurila. 1999. Genetic structure of island populations of the anurans Rana temporaria and Bufo bufo. Heredity $82: 309-317$

Silva, D.M.; D.A., Da Cruz, R.P., Bastos, M.P., De C. Telles \& J.A.F., Diniz-Filho. 2008. Morphometric and genetic differentiation among populations of Eupemphix nattereri (Amphibia, Anura, Leiuperidae) from central Brazil. Iheringia Sér. Zool. 98: 493-500.

Sinsch, U. \& H., Schneider. 1999. Taxonomic reassessment of Middle Eastern lake frogs: Morphological variation 
among populations considered as Rana ridibunda, $R$. bedriagae or R. levantina. J. Zoo. Syst. Evol. Res. 37: 67-73.

Sinsch, U., H., Schneider, U., Kaya \& H., Arikan. 2002. The water frogs (Anura: Ranidae) of Turkey: a morphometric view on systematics. Herpetol. J. 12: 141-153.

Stöck, M., A. Sicilia, N.M. Belfiore, D. Buckley, S. Lo Brutto, M. Lo Valvo \& M. Arculeo. 2008. Evolutionary relationships across the Sicilian channel: Mitochondrial and nuclear markers link a new green toad from Sicily to ancient African relatives. BMC Evol. Biol. 8: 56.

Tryjanowski, P., T., Sparks, M., Rybacki \& L., Berger. 2006. Is body size of the water frog Rana esculenta complex responding to climate change? Naturwissenschaften, 93: 110-113.

Wells, K.D. 2007. The Ecology and Behavior of Amphibians. University of Chicago, Chicago, EEUU. 
